Renata Suszczyńska

\title{
Der Einsatz der Medien im Fremdsprachenunterricht im 21. Jahrhundert am Beispiel des Deutschlektorats an der Warsaw School of Econocmics
}

Renata Suszczyńska: Médiahasználat a 21. századi idegennyelv-oktatásban a Varsói Gazdasági Főiskola Német Nyelvi Lektorátusának példája alapján

\begin{abstract}
Összefoglaló
A tanulmány néhány fontos médiapedagógiai és didaktikai kérdéssel foglalkozik. Az elsö részben a médiumok meghatározására és a médiahordozók csoportosítására vállalkozom. A második részben a médiumok nyelvoktatásban történö alkalmazásának elönyeit és hátrányait emelem ki, és közben feltevéseimet néhány, a diákok prezentációs kompetenciájának fejlesztésére irányuló gyakorlati példával illusztrálom.
\end{abstract}

Kulcsszavak: régi és új médiumok, audiovizuális tanítási módszer, a médiumok előnyei és hátrányai a nyelvoktatásban

\section{ABSTRACT}

Im Beitrag werden einige wichtige medienpädagogische und mediendidaktische Fragestellungen behandelt. Im ersten Teil werden die Definition der Medien und die Gliederung der Medienträger vorgenommen. Im zweiten Teil werden die Vor- und Nachteile des Medieneinsatzes

im

Fremdsprachenunterricht hervorgehoben und dabei einige praktische Beispiele zur Förderung der Präsentationskompetenz der Studierenden aufgeführt.

Schlüsselwörter: alte und neue Medien, audiovisuelle Lehrmethode, Vor-und Nachteile der Medien im Fremdsprachenunterricht

\section{EINLEITUNG}

Die allgegenwärtigen Medien sind aus unserem Leben nicht mehr wegzudenken. Keiner von uns kann sich das Leben ohne Presse, Fernsehen oder Radio vorstellen, immer häufiger auch ohne Computer, Tablet-PC, I-Pad und ohne Internet. Heute, wenn jeder Student mit allen digitalen Medien gut vertraut ist und praktisch die ganze Zeit ans Internet angeschlossen ist, wäre es leichtsinnig unsererseits (der Lehrer) diese Tatsache auch im Fremdsprachenunterricht nicht zu nutzen. Den neuen Medien kommt eine besondere Bedeutung zu. Mittels ihres multimedialen Charakters können sie den Fremdsprachenunterricht effektiv unterstützen. Während noch in den 80er Jahren des 20. Jahrhunderts die Lernenden einen Hörtext im Unterricht scheuten, erwarten sie heute eine Abwechslung wie einen Film oder eben einen Hörtext, und zwar einen authentischen. Das geschriebene Wort hat glücklicherweise noch nicht ganz an Bedeutung verloren, aber die heutigen Jugendlichen leben in der Epoche der audiovisuellen Errungenschaften und möchten diese auch im Unterricht nutzen.

\section{MEDIEN-DEFINITION}

Es wäre angebracht, am Anfang dieser Überlegungen von der Definition der modernen Medien auszugehen. Hans Toman [2008] gibt die folgende Definition der modernen Medien: 
„Der Terminus ,Medium" entstammt dem Lateinischen. Es bedeutet ,Mitte', Mittelglied' oder ,Mittler'. Es bezeichnet im heutigen Sprachgebrauch technische Geräte und Programme, die auf Bild-, Ton- und Datenträgern gespeichert sind. Medien dienen der Erfassung, Verarbeitung und Übermittlung von Informationen und verbreiten visuelle und akustische Botschaften."

\section{GLIEDERUNG DER MEDIEN}

Im heutigen Fremdsprachenunterricht unterscheidet man zwischen andersartigen Medienarten, die nach unterschiedlichen Aspekten eingeteilt werden können. Wir können daher von Print-, visuelle, auditive, audiovisuelle oder neue Medien sprechen.Nach einer anderen Einteilung sind es Primärmedien, Sekundärmedien und Tertiärmedien: Unter den Primärmedien versteht man alltägliche zwischenmenschliche Kommunikation, ohne Hilfe von Technik, Face to Face-Gespräche (Kommunikation). Als Sekundärmedien bezeichnet man solche Medien, die bei ihrer Produktion Hilfsmittel benötigen (Bücher, Zeitungen).Tertiärmedien bedürfen sowohl bei der Produktion, als auch bei der Rezeption technischer Hilfsmittel (CD, Video).

Die populärste Einteilung umfasst traditionelle und computerbasierte Medien [Nattland - Kerres 2006]

\section{GEGENWÄRTIGE MEDIENTRÄGER}

Abgesehen von der Klassifizierung der Medien unterscheidet man heutzutage folgende Medienträger:

- Presse (Zeitungen, Zeitschriften)

- Buch (Fachbücher, Literatur etc.)

- Rundfunk und Fernsehen

- Film (Kino, Video)

- Tonträger (Schallplatte, Tonkassette, CD, MP3 etc.)

- Computersoftware (USB-Stick, CD-ROM etc.)

- Telekommunikation (Internet, OnlineDienste, E-Mail , Telefon, Handy)

\section{PRESSE IM FREMDSPRACHENUNTERRICHT}

Seitdem Johann Gutenberg im 15. Jh. die beweglichen Lettern erfunden hat, sind die Druck-Medien die populärsten in der zwischenmenschlichen Kommunikation [Martiny 2008]. Das erste und wichtigste Medium im Unterricht ist natürlich das Lehrbuch. Im Fremdsprachenunterricht taucht jedoch die Frage auf, wie effektiv man nebenbei die Presseartikel aus den Tageszeitungen oder aus den Zeitschriften nutzen kann.

An der Wirtschaftshochschule Warschau verläuft die Arbeit mit einem Pressetext auf unterschiedliche Weise. Einmal in der Woche bereiten die Studenten der älteren Semester die sogenannte Presseschau vor. Sie suchen im Internet nach den aktuellsten zwei bis drei Nachrichten, fassen diese zusammen, um sie dann vor der Gruppe vorzutragen. Es ist üblich vor der Präsentation eine Vokabelliste zu präsentieren. Es wird auch mit längeren Presseartikeln gearbeitet, entweder im Unterricht oder zu Hause nach den vom Lehrer angegebenen Hinweisen. Es kommen vor allem solche Titel in Frage wie: "Wirtschaftswoche“, „Handelsblatt", Artikel von der Webseite "Deutsche Welle", Tageszeitungen wie „FAZ", "Die Welt" oder Magazine wie "Der Spiegel". Wir hoffen, dass dank solchen Übungen die Studenten auch in der Zukunft nach deutschsprachigen Artikeln greifen werden. Das kurzfristige Ziel, das wir uns und den Studenten setzen, ist jedoch sie auf die mündliche Prüfung vorzubereiten.

\section{ELEKTRONISCHE MEDIEN}

Elektronische Medien, die die Kommunikation im 20. Jh. revolutioniert haben, werden im 21. Jahrhudert noch intensiver genutzt werden. Die Rolle mancher alten Medien lässt im Fremdsprachenunterricht nach, wird aber nicht ganz beschränkt, ein Beispiel dafür ist der immer noch gern eingesetzte Overheadprojektor (OHP). 
Zudem wäre es interessant zu wissen, durch welche neuen Medien Fernsehen, Radio, Video oder Computer in einigen Jahren ersetzt werden können. Vielleicht durch das interaktive Whiteboard, das immer stärker an Popularität gewinnt? Die Möglichkeiten, die das Whiteboard bietet, sind umfangreich. Der Lehrer kann das Tafelbild stoppen, speichern und es wieder in einem anderen Moment zeigen und besprechen. Ein großer Nachteil der interaktiven Tafel ist ihr hoher Preis, der über finanzielle Möglichkeiten mehrerer Schulen hinausgeht [Rebensburg 2010].

Vielleicht ist die Frage nach der Ersetzung alter Medien durch neue gar unberechtigt? Da, wie die Praxis zeigt (und Faulstich in seiner Arbeit feststellt), bis jetzt kein Medium im Unterricht durch ein anderes ganz verdrängt wurde. Es kann also zu einer Situation kommen, dass im 21. Jahrhundert der Lehrer von so vielen Medien umgeben ist, dass es ihm schwer fällt, sich für das Richtige zu entscheiden. Dies aber lässt zugleich hoffen, dass der moderne Unterricht nicht langweilig sein wird.

\section{MEDIENPÄDAGOGIK}

Da der Einsatz der Medien im Unterricht immer populärer wurde, war es erforderlich einen neuen Bereich der Didaktik zu schaffen: die Medienpädagogik. Sie befasst sich mit bisher unbekannten Problemen wie z.B.vernünftige Mediennutzung. Den Lernenden wird beigebracht, wie sinnvoll sie verschiedene Medientypen nützen können, um sowohl ihre Bedürfnisse zu befriedigen, als auch ihre Fähigkeiten zu entwickeln. Neue Medien können im Fall des Fremdsprachenunterrichts wesentlich die Entwicklung der Sprachkompetenz verstärken und beeinflussen. Die Rolle des Lehrers besteht außerdem darin, den Lernenden zu zeigen, wie sie nach nötigen Informationen suchen sollen, und was besonders wichtig ist, die gefundenen Informationen auch kritisch wahrzunehmen.

\section{MEDIENKOMPETENZ}

Im engen Zusammenhang mit der Medienpädagogik steht die sogenannte
Medienkompetenz. Im heutigen und viel mehr im zukünftigen Fremdsprachenunterricht scheint gerade diese Kompetenz von großer Bedeutung zu sein. Lernende, die die Medienkompetenz beherrscht haben, sind fähig ohne Probleme mit den heutigen Informationsund Kommunikationstechnologien so perfekt umzugehen, dass sie diese erfolgreich innerhalb und außerhalb des Unterrichts nutzen können. Die Medienkompetenz beschränkt sich nicht ausschließlich auf den geschickten technischen Umgang mit neuen Medien; vielmehr soll der Lernende die Vorteile der Medien im Lernprozess nutzen können. Und gerade diese Fähigkeit versuchen wir unseren Studenten beizubringen, damit sie auch den Eindruck gewinnen, etwas Praktisches gelernt zu haben.

\section{VORTEILE UND NACHTEILE DES MEDIENEINSATZES IM FREMDSPRACHENUNTERRICHT}

Jeder Lehrer, der mit den Medien arbeitet oder arbeiten will, steht oft vor einem Dilemma: Inwieweit "lohnt es sich" die Medien im Unterricht einzusetzen? Ist es nicht vielleicht ein Zeitverlust? Können meine Studenten überhaupt davon profitieren, was ich ihnen zeige oder vorspiele?

Gestatten Sie mir an dieser Stelle die folgende Frage zu beantworten: Warum ist der Einsatz der modernen Medien von so großer Bedeutung für den Fremdsprachenunterricht im 21. Jahrhundert?

\subsection{Vorteile}

Es gibt eine Menge von Nutzen; es seien hier einige von ihnen genannt, insbesondere diejenigen, die ich selbst während meiner Arbeit beobachtet habe.

- $\quad$ Bei einem Film oder einer Fernsehreportage bekommen die Lernenden gleichzeitig Informationen über die Augen und die Ohren, so dass mehrere Sinne angesprochen werden. Das steigert ihre Konzentration und lässt das präsentierte Material länger in Erinnerung bleiben. 
- Der Lektor kann Materialien wählen, die den Bedürfnissen der Studenten entsprechen und dadurch ihre Aufmerksamkeit wecken.

- Dem Lehrer sowie den Lernenden steht dank dem Internet eine unerschöpfliche Quelle der Lehrmaterialien zur Verfügung.

- Die Lernenden bekommen authentische Informationen aus dem Land, dessen Sprache sie lernen. Das Material, das sie schon gelernt haben, können sie dank einem Film visualisieren.

- Sogar ein kurzer Film kann bei dem Verstehen oder Erarbeitung eines Themas helfen.

- Filme, Hörtexte oder Presseartikel regen die Lernenden zur Diskussion an, die umso heftiger wird, je interessanter und aktueller die berührten Themen sind.

- Der Lehrer und die Studenten können auf die Ereignisse in der Politik, Wirtschaft oder Kultur sofort reagieren und auf dem Laufenden sein.

- Wenn die Lernenden entdecken, dass sie imstande sind, authentische Texte oder Filme zu verstehen, seigt ihre Motivation zum weiteren Lernen.

- Den Lernenden wird die Medienkompetenz beigebracht.

\subsubsection{Präsentationskompetenz}

Neue Medien, insbesondere Computer und solche Programme wie Power Point oder Prezi ermöglichen es den Studenten eine neue Kompetenz, d. h. die Präsentationskompetenz zu entwickeln. An unserer Hochschule legen wir großen Wert auf die Entfaltung dieser Kompetenz.

Jeder Student bereitet alleine oder mit einem anderen Studenten einmal im Semester eine professionelle Präsentation vor. Die Thematik ist unterschiedlich, wir versuchen jedoch die Aufmerksamkeit der Studenten auf die Problematik der deutschsprachigen Länder zu lenken, was sie auch gern in ihren Referaten tun. Im Hinblick auf das wirtschaftliche Profil unserer Hochschule präsentieren die Studenten sehr oft Firmen aus dem deutschsprachigen Raum oder ihre Produkte.
Die Studenten können auf zweifache Weise von dieser Aufgabe profitieren: Erstens vertiefen sie ihr Wissen über deutschsprachige Länder, zweitens lernen sie, eine Präsentation vorzubereiten und vor der Gruppe zu halten, was in ihrer zukünftigen Arbeit von Nutzen sein kann.

Obwohl ich völlig überzeugt bin, dass die Medien im Fremdsprachenunterricht mehrere Vorteile mit sich bringen, sollte man auch die Nachteile der Mediennutzung vor Augen halten.

\subsection{Nachteile:}

- Die Arbeit mit den neuen Medien erfordert von dem Lehrer eine besonders gründliche Vorbereitung. Es reicht nicht aus, den Studenten einen Film zu zeigen (der Unterrichtsraum ist doch kein Kinosaal), der Lehrer muss den Unterricht, in dem Medien eingesetzt werden, oft länger und gründlicher konzipieren. Nicht selten didaktisiert der Lehrer selbst das dargestellte Material, Film, Reportage, etc.

- Das Überangebot der Materialien im Internet erschwert oft die richtige Wahl und nimmt wieder viel Zeit in Anspruch.

- Die meisten Materialien lassen sich hauptsächlich in fortgeschrittenen Gruppen besprechen.

- Man spürt einen starken Einfluss der englischen Sprache, was oft sogar akustisch das Verstehen des deutschen Textes erschwert, wenn im Hintergrund der englische Text läuft, wie oft auf Youtube.

- Der Einsatz von authentischen Texten oder Filmen ist im Unterricht für Anfänger manchmal wenig produktiv. Es können zwar kurze, leichte Filme gezeigt, oder Hörtexte vorgespielt werden, die vor allem der Vorstellung über das Leben im Land der Sprache dienen, sie können aber auch demotivierend auf die Lernenden wirken, wenn diese wenig oder kaum etwas davon verstehen.

- Manche Lehrer haben Angst vor dem Computer, weil sie denken, sie sind technisch nicht genug begabt, um mit dem Computer umzugehen. Es fehlt an den 
Renata Suszczyńska: Der Einsatz der Medien im Fremdsprachenunterricht im 21. Jahrhundert ...

Schulen also eine Form der Schulung, die solche Mängel beheben könnte.

- Beim Einsatz solcher Medien, wie das Internet, im Unterricht ist das Haupthindernis der Preis. Nicht alle Schulräume sind so modern ausgestattet, dass man den Unterricht auf Basis des Internetanschlusses führen kann.

\section{ZUSAMMENFASSUNG}

Zusammenfassend möchte ich Folgendes betonen: Der moderne Lehrer im 21. Jahrhundert darf die neuen Medien nicht scheuen. Er muss ihre Bedienung dermaßen beherrschen, dass sie seinen Unterricht begleiten und effektiv unterstützen kann.
Heutzutage darf der Lehrer den Fremdsprachenunterricht nicht nur auf das Lehrbuch und die Tafel beschränken. Es lässt sich nicht anzweifeln, dass vernünftig angewandte Medien den Lerneffekt wesentlich steigern. Das breite Spektrum verschiedener Medienträger wie Handys, E-Books, Tablet-PCs, MP-3 Player und viele andere mehr, eröffnen vor den Lehrern und Lernenden unbegrenzte Möglichkeiten. Der Lernende kann praktisch jederzeit eine Aufnahme z.B. auf seinem Handy hören und auf diese Weise während der Fahrt zur Schule das Hörverstehen üben. Obwohl sie sich so rasant entwickeln, bleibt uns doch zu hoffen, dass der Lehrer durch die neuen Medien in der Zukunft nicht ganz ausgeschaltet wird.

\section{BIBLIOGRAPHIE}

[1] Martiny, K. (2008): Der Einsatz von Neuen Medien im Fremdsprachenunterricht am Beispiel einer Blended-Learning-Unterrichtseinheit für das Fach Englisch in der Sekundarstufe. Technische Universität Darmstadt.

http://www.daf.tudarmstadt.de/media/daf/dateien/pdfs/studienarbeiten/martiny_persn lichedatenentfernt.pdf (Zugang: 11.10.2014)

[2] Nattland, A. - Kerres M. (2006): Computerbasierte Medien im Unterricht.In: K. -H. Arndold. U. Sandfuchs - J. Wiechmann (Hrsg.) Handbuch Unterricht. Bad Heilbrunn.

[3] Rebensburg, K. (2010), Medien im Unterricht, Was gab es? Was gibt es? Was wird es geben? Universität Potsdam http://fue-wiki.tubit.tuberlin.de/lib/exe/fetch.php/lehrveranstaltungen:leitbilder:ausarbeitung_sandy_roigk.pdf (Zugang: 11.10.2014)

[4] Toman, H. (2008), Medien im Unterricht. http://www.dr-toman.de/Medpaed08Langversion.pdf (Zugang: 15.10.2014) 